rev.relac.int.estrateg.segur.9(1):87-110,2014

\title{
LA TEORÍA DEL ESTADO FALLIDO: ENTRE APROXIMACIONES Y DISENSOS•
}

\author{
John Sebastián Zapata Callejas ${ }^{* *}$
}

\section{RESUMEN}

Debido a la proliferación de investigaciones realizadas en las dos últimas décadas acerca de la teoría del Estado fallido a nivel global, se hace pertinente comenzar a analizar la literatura especializada que se ha producido en habla hispana. Con base en lo anterior y teniendo en cuenta que no existen consensos alrededor de la noción de Estado fallido, el presente artículo tiene como objetivo elaborar una reconstrucción teórica de diversos textos en español, que aluden a dicha temática. Para tal fin este escrito se divide en tres momentos: en el primero se desarrolla el origen del término, las principales definiciones, las escuelas o corrientes y algunos conceptos relevantes en relación a la falla estatal; un segundo momento evidencia el debate metodológico

* Este artículo es resultado parcial del proyecto de investigación "Las transformaciones contemporáneas del Estado, la seguridad y los conflictos: un estado del arte sobre la construcción de paz y la paz liberal", financiado por el CODI (2012-2013) y desarrollado por el Grupo de Investigación de Filosofía Política de la Universidad de Antioquia. Además, el presente artículo es una versión resumida de mi tesis para optar para el título de Politólogo en la Universidad de Antioquia.

**Politólogo de la Universidad de Antioquia. zapata0514@hotmail.com 
que se da gracias a la proliferación de índices, y se muestran algunos estudios de caso; el tercer momento articula y problematiza la intervención de la comunidad internacional en los Estados fallidos.

Palabras clave: Estado fallido, falla estatal, Estado, intervención internacional.

\title{
THE THEORY OF STATE FAILURE: AMONG APROXIMATIONS AND DISAGREEMENTS
}

\begin{abstract}
Due to the global proliferation of research regarding the theory of State failure in the last two decades, it becomes relevant to start analyzing the applicable literature that has been produced in Spanish. Based on this, and bearing in mind that there is no consensus on the notion of a failed State, this article will develop a theoretical reconstruction of several Spanish texts that refer to that topic. To this end, this paper is divided into three stages: the first time when it develops, the origin of the term, the main definitions, the schools or trends and some important concepts related to State failure. A second time to show the methodological debate that surges thanks to the proliferation of indices, and similarly, show some case studies. Last, a third moment that articulates and show the problems due to the international community's intervention in failed States
\end{abstract}

Keywords: Failed State, state failure, State, international intervention.

\section{TEORIA DO ESTADO FALIDO: ENTRE APROXIMAÇÕES E DISCÓRDIAS}

\section{RESUMO}

Devido à proliferação das pesquisas realizadas sobre a teoria do Estado falido no âmbito global nas últimas duas décadas, torna-se pertinente começar a analisar a literatura especializada que tem sido produzida na língua espanhola. Com respeito ao anterior e levando em consideração que não existe um consenso em torno do conceito de Estado falhado, o objetivo deste artigo é o de elaborar uma reconstrução teórica de vários textos em espanhol que se referem a esse tópico. Para este fim, este trabalho está dividido em três etapas: a primeira, na qual se aborda a origem do termo, as definições principais, as escolas ou correntes e alguns conceitos relevantes relacionados com a falência estatal; a segunda, que evidencia o debate metodológico que ocorre graças à proliferação de índices, e da mesma forma, mostra alguns estudos de caso; e uma terceira etapa, que articula e problematiza a intervenção da comunidade internacional nos Estados falidos.

Palavras-chave: Estado falido, falha estatal, Estado, intervenção internacional 


\section{ORIGEN DEL ESTADO FALLIDO}

El concepto de Estado fallido es una idea contemporánea que da cuenta básicamente de las problemáticas, deficiencias e imposibilidad de ciertos Estados para responder a las diversas demandas que hacen sus ciudadanos. Serían los internacionalistas Herman y Rartner, a principios de la década de los años noventa, los primeros en utilizar el concepto de Estado fallido. Esos analistas se preocupaban por un nuevo modelo a través del cual un Estado llegaba a ser totalmente incapaz de mantenerse como un miembro de la comunidad internacional. Según esos autores un Estado fallido pondría en peligro a sus propios ciudadanos y amenazaría a sus Estados vecinos, a causa del flujo de refugiados, inestabilidad política, conflictos, revoluciones y demás (Di John, 2010, p. 49). A esta primera definición, se le han sumado otras teorías que han generado que el concepto de Estado fallido sea un término, que si bien se utiliza y legitima constantemente en el campo político, mediático y académico, también posee un gran número de críticas y debates.

En lo que respecta al campo académico es propio recordar que a las definiciones, causas y consecuencias de la falla estatal, suele dársele un enfoque que privilegia alguna ciencia social; ya sea la ciencia política, la economía, la antropología, el derecho, la sociología, las relaciones internacionales y demás. Es decir, que dependiendo del campo de conocimiento desde el que se avanza o se privilegia, el análisis de la falla estatal da prioridad a distintos tipos de tesis e indicadores; por ejemplo, un economista no duda en considerar el ingreso per cápita, la inflación, la tasa de desempleo o el índice de desarrollo humano como indicadores críticos, que pueden originar o aumentar la falla estatal (Flórez, 2011).

A pesar de la existencia e importancia de los diversos enfoques que se pueden utilizar para analizar la teoría de la falla estatal, se debe advertir que es menester estudiar dichos enfoques de manera conjunta para no caer en ópticas unidimensionales que no abarquen como tal todas las dinámicas del objeto de estudio-en este caso el Estado- ya que tanto las lógicas políticas, económicas, internacionales, jurídicas, sociales y culturales producen Estados fallidos.

Del mismo modo, a la hora de hablar de Estados fallidos, se debe recordar el problema de la sinonimia o proliferación semántica, ya que se observa que cuando las diferentes comunidades políticas, académicas y periodísticas trabajan el Estado fallido, lo hacen de manera indiscriminada en relación a términos como el Estado premoderno, el Estados frágil, los Casi Estados, el Estado criminal, los Estados desestructurados, el Estado cómplice, los Estados débiles, para mencionar algunos casos. Sin embargo, y según Pere Vilanova,

“El nexo común a todos estados conceptos (y otros que están por venir), es el concepto de estado, pero en todos los casos, vinculado a alguna anomalía: sistemas políticos que o bien son formalmente estados, pero no consiguen desarrollar con estándares de normalidad las funciones propias de tal condición, ni "hacia dentro" (soberanía interna) ni hacia afuera (en relación al concepto de estado soberano en el plano internacional" (Vilanova, 2008, p.3). 
Pese que al compararlos con la noción de falla estatal pueda existir algún parecido terminológico entre los anteriores conceptos y de igual manera coincidencia en cuanto a que todos tienen anomalías en su sistema político, se debe reconocer que el Estado fallido es una relevante perspectiva de análisis que se viene desarrollando posterior a la Guerra Fría y que posee un cúmulo académico bastante amplio (autores especializados, centros de estudios, escuelas, etc.) y por lo mismo, se debe apreciar como una teoría propia en las tipologías estatales.

\subsection{DEFINICIONES}

Conociendo que la teoría del Estado fallido se originó en los años noventa, es importante explorar algunas definiciones significativas de este concepto que fueron construidas por pensadores o instituciones relevantes en el campo de las relaciones internacionales y ciencias sociales.

Para Jean Marie Grose existen cinco tipos de Estados fallidos:

1. Anarchicstates; donde no hay poder político centralizado.

2. Phantomstates; sólo hay una autoridad limitada de Estado.

3. Anemicstates; Estados con escasos recursos que se encuentran en guerra contra grupos secesionistas.

4. Capturedstates; Estados que están manejados por grupos étnicos.

5. Abortedstates; no hay poder único que posea el monopolio de la fuerza (Álvarez, 2007, p. 3).

Por su parte, en 1999, Daniel Thürer trabajó el failed state o Estado fallido, llegando a la conclusión que ese término era muy impreciso y lo sustituyó por desintegrated o collapsed states, sobre los que hace una triple aproximación conceptual: política, histórica y sociológica (Álvarez, 2007, p. 3). Según Rainer Tezlaff, la noción de fracaso estatal es un asunto multidimensional a largo plazo, donde el colapso del Estado es el punto final de dicho proceso (Emeka, 2007, p. 2). En 1998, la Cruz Roja teorizó el Estado fallido, pero lo hizo desde la perspectiva de la desestructuración de los conflictos armados, es decir, un tipo de Estado que pasa a ser apropiado por una variedad de actores en luchas entre sí (Álvarez, 2007, p. 4).

Otro de los autores que ha venido trabajando la teoría de la falla estatal es Robert Jackson. Según él, los Estados fallidos son los Estados que se han autodestruido gracias a la anarquía armada que impera al interior de sus fronteras, lo que ha llevado, por ejemplo, a que su gobierno falle o los ciudadanos fallen a su gobierno, es decir, que no exista una institución política que ponga en ejercicio su poder. En otras palabras, plantea que los Estados fallidos son los que no son capaces de salvaguardar las condiciones civiles mínimas para sus conciudadanos (paz interna, leyes, orden, buen gobierno, etc.); Estados, que si bien tienen un reconocimiento legal 
internacional que los identifica como Estados-Nacionales soberanos, dentro de sus fronteras la legalidad y su potestad de aparato regulador, se difumina (Jackson, 2008, p. 5).

Por otro lado, el internacionalista especializado en Estados fallidos Robert Rotberg, postula que los Estados se encuentran básicamente diseñados para proveer una serie de bienes políticos a sus ciudadanos, los cuales son: una adecuada infraestructura de telecomunicaciones; un sistema financiero y fiscal oportuno; igualmente, el Estado tiene que dar a las personas seguridad, un sistema jurídico y judicial, libertades políticas, derechos económicos, sociales y culturales (Moncada, 2007, p. 95); y por lo mismo, se puede establecer que un Estado falla o fracasa cuando llega a incumplir dichos bienes políticos. En palabras de Rotberg: "el Estado fallido [es el que] no tiene la capacidad o la voluntad necesarias para desempeñar las funciones que le competen" (Moncada, 2007, p. 101). Se debe mencionar que el autor hace una importante clasificación de tipologías estatales, mostrando genéricamente, cuatro tipos de Estados: el fuerte, el débil, el fracasado y el colapsado .El Estado fallido es un grado entre el Estado colapsado y el Estado fracasado (Moncada, 2007, p. 95).

Dentro de los postulados de Rotberg con relación al Estado fallido, se encuentra que los mismos parten de un supuesto y tres tesis. El supuesto es que el Estado moderno occidental es un condicional dado e incuestionable; de la misma manera, describe que la primera tesis es que la causa de la falla estatal es producto de la mano del hombre; la segunda es que la falla estatal o fracaso estatal es evitable y no es irreversible y, finalmente, admite que la intervención internacional tiene cimientos legítimos, ya que en un mundo interconectado los Estados fallidos no pueden aislarse del resto de la comunidad internacional (Moncada, 2007, pp. 96-97).

Frente a esta amalgama de definiciones, se hace pertinente mostrar otras posiciones teóricas del Estado fallido que difieren, por ejemplo de la línea Rotberg, a la que se considera conservadora, y que definen el Estado fallido desde una perspectiva más crítica; Aquí se encuentran autores como: Jefrey Herbst, Christopher Clapham, Susan Woodward, Mark Duffield y en parte Noam Chomsky. Si bien es cierto que éste último escritor no se centra en realizar una amplia teorización de los Estados fallidos, se puede observar en él una tesis sobre la falla estatal, de tinte crítico y particular, debido a que propone que:

“Entre las prioridades más características de los Estados fallidos figura el que no protegen a sus ciudadanos de la violencia -y tal vez inclusión de la destrucción- o que quienes toman las decisiones otorgan a esas inquietudes una prioridad inferior a la del poder y la riqueza a corto plazo de los sectores dominantes del Estado. Otra característica de los Estados fallidos es que son estados forajidos, cuyas cúpulas se desentiende con desdén del derecho y los tratados internacionales" (Chomsky, 2007, p. 49).

La definición de Chomsky apunta a que los Estados fallidos son aquellos que tienen la particularidad de que no protegen a sus ciudadanos e, igualmente, no respetan el derecho internacional, por lo que pueden interferir y violar los derechos de otros Estados. 
Por su parte, Jefrey Herbst establece respecto, a la falla estatal, que la misma es un común denominador a través de la historia y casi inherente a la idea de Estado. Es por ello que en la actualidad se puede constatar en diferentes países la existencia de varios Estados fallidos, debido a que un gran número de Estados cuentan con complejas problemáticas dentro de sus fronteras.

En sus lineamientos, Herbst muestra que en el siglo XX se creó un imaginario de que "tener una organización política a gran escala seguía siendo difícil, pero ya no había vecinos amenazantes que tuvieran la motivación de ocupar el territorio contiguo si surgían problemas" (Moncada, 2007, p. 286).Como consecuencia se idealizó la noción de Estado Nación, y se estudiaron poco las fallas internas desde la lógica de las relaciones internacionales; sin embargo, para el autor: "el Estado-Nación, tal y como se teoriza en la actualidad, no es significativamente distinto de lo era hace 100 años" (Herbst, 2007, p. 288); porque tiene los mismos problemas. En resumen, para Herbst existen una serie de observaciones que se pueden destacar sobre los Estados fallidos o fracasados: los Estados fracasan dentro de sus fronteras; hay pocos incentivos para los actores locales para que éstos ayuden a restablecer el orden; existen fuertes presiones para que el poder continúe fracturándose en los Estados fallidos, y, el fracaso del Estado probablemente se extenderá más allá de los límites territoriales de un Estado concreto (Herbst, 2007, pp. 288-292).

De otro lado, Christopher Clapham parte de que "en los últimos tiempos se ha demostrado que los requisitos de ingeniería social sobre los cuales descansa el proyecto de estatalidad universal pueden ser en gran medida excesivos e incluso contraproducentes" (Clapham, 2007, p. 270), y cree firmemente, que si bien se han dado casos exitosos donde se ha importado el modelo de Estado occidental, en otros lugares del globo ese proceso ha sido difícil y hasta desastroso, por lo que relativiza el éxito y la viabilidad del modelo de Estado occidental.

En definitiva, para Clapham la falla estatal está directamente relacionada con la idea misma de Estado-Nación que se concibe en la modernidad. Por ello establece que:

“El problema de los Estados Fallidos consiste, sobre todo y fundamentalmente, en injertar esos Estados [...] en raíces no muy prometedoras [...] aun si se recurre a los distintos tipos de fertilizantes proporcionados por el sistema internacional bajo la forma de ideologías universalistas, la incorporación de esos Estados a la economía global y el suministro de apoyo diplomático y militar" (Clapham, 2007, p. 242).

Para Susan Woodward y Mark Duffield, la idea de Estado fallido está marcada por un ideario discursivo particular de ciertos actores en la escena internacional. Woodward dice que "el concepto es vago, incluso tautológico [y] se ha convertido en una etiqueta de moda y en un cajón de sastre, demasiado impreciso para llevar a cabo un análisis de sus causas o consecuencias y, por lo tanto, susceptible de ser utilizado de un modo inadecuado" (Woodward, 2005, p. 7). Para Duffield: "El Estado fallido es la idea que antecede al actual discurso de los Estados frágiles; 
pero que tienen en común que simbolizan, desde el punto de vista político y académico, la antítesis de un Estado eficiente, eficaz o fuerte, y donde predominan lógicas: de exclusión, pobreza, desigualdad, poca soberanía, violencia, entre otras" (Duffield, 2008, p. 3).

En últimas, autores como Daniel Thürer, William Zartman, Noam Chomsky, Christopher Clapham, Robert Rorberg, Susan Woodward, Rainer Tezlaff, Mark Duffield y Jefrey Herbst, si bien discrepan en muchas cuestiones teóricas, igualmente tienen puntos en común con respecto a las condiciones para denotar a un Estado como fallido. Entre ellas se encuentran: la ineficacia en prestar seguridad tanto interna como externa (pérdida del monopolio de la fuerza); la incapacidad para prestar servicios básicos a los ciudadanos; inestabilidad institucional; alta corrupción; crisis económicas; deficiencias jurídicas; luchas culturales; inseguridad constante, entre otras.

De hecho, a pesar de las ambigüedades y discrepancias terminológicas, Juan Gabriel Tokatlian afirma que "La bibliografía de Estados Fallidos [comparte] una serie de denominadores comunes. Independientemente de la definición específica usada, la mayoría de los trabajos [coincide] implícitamente en marcar tres brechas entre un Estado aparente y otro empírico: una brecha de legitimidad, otra de capacidad y una última de soberanía" (Tokatlian, 2008, p. 74). Eso significa que un Estado fallido carece de un poder institucionalizado y legítimo; además es incapaz de responder a las demandas de la ciudadanía, y por lo mismo, no puede brindarle todos los bienes políticos, económicos y sociales a los individuos que conviven en el mismo.

\subsubsection{Escuelas}

Teniendo presente las definiciones mencionadas, se puede comprender que no hay un consenso respecto a la idea de lo que es un Estado fallido, sino más bien una diversidad de respuestas. Sin embargo, las mismas se pueden agrupar, respecto a la tendencia teórica en dos escuelas:

La primera escuela es la que Patricia Moncada llama la escuela conservadora de los Estados fallidos, y defiende básicamente tres tesis. La primera tesis postula que el Estado moderno occidental es la organización de poder político más adecuada y, por eso, las investigaciones sobre los Estados fallidos deben partir de que el Estado moderno occidental hay que tomarlo como dado. La segunda tesis, cosiste en que la causa directa y prácticamente exclusiva de la debilidad, falla y colapso estatal es la agencia humana o la mano del hombre, es decir, el pésimo desempeño de sus gobernantes. La tercera tesis, es que el desarrollo económico nacional e internacional se consigue mediante la liberalización del comercio y la reducción del tamaño del Estado (Moncada, 2007, p. 37).

Contrapuesta a esta tendencia, encontramos la escuela que esta misma autora denomina la escuela crítica de los Estados fallidos, la cual básicamente cuestiona los principios de la escuela conservadora, y se pregunta hasta qué punto la teoría de la falla estatal es viable para entender ciertos Estados con problemas internos. Puntualmente, la escuela crítica: 
“Defiende que hay que mantener abiertas las siguientes preguntas: ¿El Estado moderno occidental debe ser la única forma de organización del poder político?, y ¿Las causas del fracaso de los Estados son eminentemente humanas o algunas de sus características del sistema internacional como el fin de la guerra fría, el orden político y económico internacional -y los poderes que lo han instaurado-, caracterizado por la liberalización de los mercados y la reducción de las capacidades del Estado, también son responsables del problema?" (Moncada, 2007, p. 38).

\subsection{LA IDEA DE ESTADO}

El Estado es un concepto de larga data que ha sido utilizado en diversos contextos de manera diferente, y por ende ha estado acompañado de un sin número de adjetivos para especificar un determinado tipo o clase de Estado; ejemplo de tales adjetivos son: Estado liberal, Estado interventor, Estado fallido, Estado contemporáneo. Sin embargo, el uso tradicional que se le da al término de Estado viene enmarcado por la tradición contemporánea de Max Webber. Para éste sociólogo "el Estado es aquella comunidad humana que, dentro de un territorio -el territorio es un elemento distintivo-, reclama para sí (con éxito) el monopolio de la violencia física legítima" (Weber, 1992, p. 88).

Ahora bien, una de las principales críticas a la teoría del Estado fallido es que la misma parte de la concepción de Weber sobre el Estado, al igual que del nacionalismo metodológico, desconociendo con ello las lógicas contemporáneas de la globalización.

En el actual estado de cosas, que la teoría de la falla estatal se ancle en la idea clásica de Estado-Nación, hace que se dé una "vaguedad en la noción de estado fallido en buena medida porque al limitarse a ser una definición negativa en relación con el concepto moderno del Estado, da por sentado aquello que constituye el núcleo mismo del problema: la noción misma de estatalidad" (Rojas, 2007, p. 76).Es decir, plantear la problemática de los Estados fallidos sin cuestionar la misma idea de Estado de la cual se parte, es analizar dicha situación a medias porque debe pensarse la función, la capacidad y el alcance de la estatalidad weberiana, en medio de un mundo cultural, económica, política y jurídicamente conectado por la interdependencia de la globalización.

Es así, como a la hora de tratar el tema de los Estados fallidos, para algunos autores y corrientes como la escuela crítica, no es propicio tratar de volver a la normalidad estatal que se plantea en la lectura clásica del Estado liberal occidental; en otras palabras, no se puede pretender restaurar las dinámicas de los Estados fallidos, desde los cánones del Estado weberiano, ya que la reconstitución de lo estatal es más bien una transformación sustancial del Estado, por lo que se pueden explorar nuevos términos de adaptación dinámica y contingente a las lógicas de la globalización (Rojas, 2007, p. 85).

Ahora bien, problematizar el rol del Estado (en cuanto al Estado fallido), lleva a considerar nociones alternativas para la construcción del modelo político en cuestión o preocuparse por 
qué tipos de Estados pueden dar solución a una situación de fracaso estatal. Es así como surgen, por ejemplo, los Estados híbridos, en donde se trata de articular las ideas estatales modernas con el reconocimiento de prácticas particulares de los Estados, para producir algo similar a un gobierno conjunto, luego de que efectivamente un territorio haya sufrido un fracaso estatal, ya que "a pesar de que se le considera el fin de todo, del colapso [o fracaso] estatal pueden surgir procesos de reconstrucción que sigan patrones de desintegración pero también, de forma imperceptible, nuevos procesos de formación estatal" (Doornbos, 2011, p. 159).

Teniendo presente las actuales lógicas de la globalización y la posibilidad de construir o reconstruir Estados fallidos no solo dentro de los parámetros del Estado occidental, se debe advertir que aún el Estado Weberiano sigue siendo fundamental en el sistema global, ya que hasta el día de hoy, el actual sistema de Estados tiene como referente el Estado- Nación. Es por eso que la teoría del Estado fallido, si bien puede seguir partiendo del concepto tradicional de Estado, debe tener en cuenta que hay nuevas dinámicas en las relaciones internacionales y que el modelo weberiano no es el único modelo existente.

\subsection{DESARROLLO Y GOBERNABILIDAD}

Al igual que la noción de Estado, dos términos algo polémicos, pero que se han vuelto comunes a la hora de hablar de los Estados fallidos, son los conceptos de desarrollo y gobernabilidad, ya que por lo regular, se parte del imaginario de que un Estado fallido presenta casi siempre bajos niveles de desarrollo y una nula gobernabilidad. De hecho, tal imaginario ha llevado a que se consolide la lógica de que una mala gobernabilidad conduce a que no se promueva el desarrollo económico (Fernández, 2009, p. 78).

Así entonces, los Estados fallidos adoptan un común denominador de ingobernabilidad, ya que se quiebra la legitimidad que tiene la ciudadanía hacia gobierno, y éste demuestra un nulo accionar institucional, dejando de ejercer el monopolio legítimo de la violencia (Salas, 2011, p. 10), lo que desde el paradigma occidental, imposibilita el desarrollo porque el desarrollo es imposible sin estabilidad política, social y económica.

Lo problemático es que para alcanzar dicha estabilidad, se necesita entre otras cosas, un adecuado desarrollo económico y de infraestructura, por lo que en última instancia, en los Estados fallidos se consolida una circularidad entre subdesarrollo e ingobernabilidad (Fernández, 2009, p. 81), debido a que para salir del subdesarrollo se necesita de una adecuada gobernabilidad; pero para establecer un eficaz accionar de gobierno, se requiere de ciertos niveles de desarrollo. Lo anterior conlleva a que los Estados que sufren de falla estatal, queden atrapados en una ingobernabilidad y subdesarrollo interno, y posean constantes exigencias de la comunidad internacional, para mejorar, al menos, sus dinámicas de desarrollo.

Respecto al desarrollo en los Estados fallidos, Javier Alcalde creé que uno de los factores externos que han propiciado el fenómeno de la falla estatal es la influencia que ha ejercido el gran paradigma del desarrollo económico y social construido y promovido por las potencias 
industriales, para su aplicación en el tercer mundo (Alcalde, 2004, p. 12), ello, ya que más que buscar mejorar las dinámicas internas de los países tercermundistas, se han proyectado políticas mundiales para favorecer a los países más poderosos.

Con base en ese autor, se hace necesario pensar en la verdadera conexión entre desarrollo ortodoxo-promovido por la institucionalidad occidental y las necesidades de los Estados fallidos- debido a que no necesariamente los países que cuentan con fallas, deben tener como referente exclusivo el mejoramiento en los índices de desarrollo económico, sino que además se debe abogar por un mejoramiento en la gobernabilidad. De igual manera, se debe poner en tela de juicio la relación causal entre subdesarrollo e ingobernabilidad, ya que si bien es cierto que para algunos Estados se cumple tal regla, en otros se hace relativa porque unos países pueden poseer subdesarrollo, pero contar con altos márgenes de gobernabilidad o tener una constante ingobernabilidad, pero tener un desarrollo constante.

\section{METODOlOGía EN LA TEORÍA DE LA FALLA ESTATAL}

Respecto a la metodología que se usa -por lo regular- para determinar qué Estados son fallidos, se puede ver que el método cuantitativo es el preponderante; no en vano hay una tendencia, desde hace varios años en Estados Unidos y en varias capitales europeas, de un afán cuantificador consistente en identificar un conjunto de variables probables en forma de índices, para localizar exactamente el nivel de falla estatal de ciertos Estados, y a cuáles efectivamente se les podría brindar la categoría de Estados fallidos (Tokatlian, 2008, p. 73). Sin embargo, y a pesar de la proliferación de diversos índices de Estados fallidos, se puede observar que en la literatura especializada han aparecido algunos estudios que utilizan metodologías cualitativas (como el estudio de caso), para describir la situación de fracaso de Estados como Somalia, Sudan, y Timor Oriental o la relación de la teoría del Estado fallido con países como Argentina, Colombia, Nigeria, Guatemala y hasta la misma región suramericana.

\section{1 ÍNDICES}

En cuanto a los diversos índices de Estados fallidos que han surgido en la última década, vale la pena aclarar que el excesivo interés de cuantificación, ha traído algunos inconvenientes para la actual teoría de la falla estatal, en palabras de Luis Freddyur Tovar:

"el problema en este punto radica, a mi modo de entender la teoría, en que con ella se construye una generalización ahistórica y autocrática [...], implica medirlos a todos con el mismo rasero, cuando la sociedad mundial ha tenido distintos momentos de desarrollo. En efecto, no es lo mismo el grado de desarrollos de muchos Estados africanos, asiáticos y americanos, respecto de Europa o de los Estados Unidos de América. Sostener entonces, que los índices se basan en generalizaciones ahistóricas de los Estados constituye una falencia metodológica y una falacia de principio poco o nada plausible, que no se sostiene por si sola" (Tovar, 2009, p. 71). 
Es decir, esos índices e indicadores que son en su mayoría los que se toman para determinar y señalar a los Estados fallidos, han olvidado brindar estudios más descriptivos con base en metodologías cualitativas o el uso de metodologías mixtas.

En su perspectiva crítica acerca de los índices de Estado fallido, el profesor Francisco Gutiérrez Sanín plantea dos cuestiones esenciales que evidencian los problemas que enfrentan los índices de Estados fallidos: en la primera admite que si bien existe una enorme literatura sobre el Estado y su génesis, hay que indagar qué puede ser operable de ese cúmulo de información y bajo qué criterios; debido a que en los índices no queda muy claro. Para el segundo asunto, Gutiérrez Sanín establece que las calificaciones de los Estados fallidos suelen hacerle entorno a diccionarios mal formados, donde un término complejo o difuso se apoya en otro concepto igualmente impreciso, generando con ello que los índices tengan resultados poco confiables (Gutiérrez, 2009, p. 89).

De otra parte, actualmente existe un amplio número de índices que buscan clasificar a los Estados y determinar cuáles de ellos son Estados fallidos. Algunos de ellos son: el de la CIA "Political Inestability Task Force"; el de la USAID "Fragilestatesstrategy"; el "Failedstatesindex" de la Foreing Policy y el fondo para la paz; el Failed y fragile States Country Indicator de la Carton Univiersity de Canadá.

Es importante indicar que las instituciones que realizan los índices mencionados, son reconocidos centros de estudio o gubernamentales a nivel mundial, y con el pasar del tiempo y el aumento del uso de la categoría de Estados fallido en el medio académico y político, se ha producido un fenómeno donde otras instituciones de gran importancia han comenzado a hacer -en los últimos años- sus propios índices; por lo que Gutiérrez Sanín afirma que "el interés por el fenómeno ha derivado en una eclosión de ejercicios cuantitativos, [pero], por desgracia, cada autor-agencia internacional tiende a utilizar su propia nomenclatura: colapso, fragilidad, debilidad" (Gutiérrez, 2009, p. 91). Así entonces, puede establecerse que el poco consenso en la nomenclatura es una cuestión relevante que dificulta un análisis objetivo y en conjunto de todos estos indicadores e índices para tener una noción de cuáles y en qué grado los Estados se encuentran sumergidos en la falla estatal.

Otro punto polémico que se puede constatar en los índices de Estados fallidos es que

"pese a que existe un número significativo de trabajos que proporcionan una clasificación razonable y una verificación empírica de la falla estatal, hay una gran variedad en torno a las variables que miden el grado de debilidad estatal. Así [por ejemplo] el Banco Mundial ha identificado cerca de 30 países de bajos ingresos bajo presión, el Departamento británico para el desarrollo Internacional señala 46 estados frágiles, mientras que la CIA reporta en 20 los Estados Fallidos" (Rojas, 2007, p.76).

Lo anterior tiene como resultado un confuso análisis de los diversos índices, ya que cada uno tiene sus propios indicadores y variables, y por lo mismo, se llegan a establecer diversos 
resultados. Así, unos índices muestran un número de Estados fallidos, mientras que otros dan números totalmente diferentes. Sobre el tema, Diana Rojas indica que "la disparidad en los métodos de medición y sobretodo la divergencia acerca de los criterios principales para conceptualizar la falla estatal hacen que aún esté pendiente un diagnóstico más acertado y operativo que sirva tanto para el análisis como para la adopción de políticas que resultan el deterioro estatal" (Rojas, 2007, p. 77).

En cuanto a la complejidad de las variables, José Fernando Flórez en su crítica metodológica a los índices de Estados fallidos, postula que "la manera como se transforman conceptos en [variables] cuantitativas, o cualidades en números, es necesariamente problemática y deficiente por cuanto toca los límites mismos de las ciencias sociales y su capacidad explicativa" (Flórez, 2011, p. 199). Entonces, al igual que como sucede en otras teorías y conceptos de las ciencias sociales, persiste la pregunta de cómo abordar los Estados fallidos en relación con sus cuestiones cuantitativas y cualitativas; es decir qué se puede contar y qué se puede cualificar.

Con base en los anteriores conceptos, es posible establecer que las principales críticas a los índices de Estados fallidos se centran en las generalizaciones ahistóricas de las que parten; sus problemas en la nomenclatura; las divergencias de variables entre los diferentes índices; y la misma transformación de conceptos cualitativos a variables cuantitativas.

Para finalizar, es apropiado establecer que la disyuntiva que poseen los índices de Estados fallidos no se puede determinar en términos dramáticos y fatalistas; simplemente se puede llegar a la conclusión de que los índices acerca de la falla estatal son tanto difíciles de realizar como a su vez, son deseables para poder tener un amplio conocimiento de los Estados fallidos (Gutiérrez, 2009, p. 101). De igual manera, no se puede descartar el uso de ningún método, ya sea el cualitativo, el cuantitativo o el mixto, debido a que cada uno cuenta con su propia utilidad; y el uso de cada método varía dependiendo de las características y necesidades del objeto estudiado.

\subsection{ESTUDIOS DE CASO}

En los escritos que sobre los Estados fallidos se han producido en español, se pueden observar algunos estudios de caso sobre ciertos países que poseen el adjetivo de fallidos. En ocasiones, dichos estudios coinciden con el dictamen de los diferentes índices, así como con el discurso de ciertas comunidades académicas, instituciones internacionales y entes políticos, sobre cuáles países están o pudiesen estar en situaciones de fracaso o de falla.

Así, en la serie de estudios de caso, se puede observar una lista encabezada por Somalia, paradigma de Estado fallido durante los últimos veinte años. Ese país ha tenido que afrontar una serie de catástrofes a nivel político, económico, cultural y social, que han llevado a un fracaso total a su aparato estatal. De hecho, "desde hace más de veinte años el país se encuentra envuelto en un conflicto interno que ha causado más de 350.000 bajas y producido alrededor 
de un 1,5 millones de desplazados internos" (Castellon, 2011, p. 137). Esa situación ha Ilevado a ese país del cuerno de África a una fragmentación territorial; "del antiguo territorio Somalí, han emergido Somalilandia, Somalia y Putlandia. La ex Somalia italiana (hoy Somalia) se ha fragmentado en un sin número de pequeños Estados independientes, e incluso Mogadiscio se ha divido en pequeños Estados Clánicos" (López \& Alzamán, 2010, p. 585).

Para solucionar la situación de Somalia, y teniendo presente el coste internacional del fenómeno de la piratería Somalí para la comunidad internacional, se han elaborado una serie de intervenciones tanto regionales como internacionales. Sin embargo, sus efectos han sido relativos y hoy en día, Somalia sigue teniendo los mismos problemas territoriales, sociales, políticos y económicos.

De igual manera, se pueden encontrar casos complejos como el de Sudán, Timor Oriental y Pakistán. Para el primer caso, cabe recordar que Sudán se ha establecido como Estado- Nación mediante exclusión constante de ciertas regiones del país (en especial la del sur), ya que las élites del norte han querido establecer un poder hegemónico anclado a un paradigma cultural radical en pro de ideas árabes-musulmanas, generando con ello una serie de conflictos. Según Aleksi Ylönen "este proyecto ha convertido Sudán a un estado fallido para los grupos de la periferia en el sur, el este y el oeste, condenándolos junto con sus regiones a un subdesarrollo persistente y a la falta de crecimiento económico, produciendo agravios en el origen de los conflictos regionales que el conflicto sudanés ha intentado sofocar continuamente desde 1955" (Ylönen, 2008, pp. 20-21). No en vano, el conflicto interno, la violencia militar, las guerras civiles, las crisis humanitarias y los bajos índices de desarrollo humano, son prueba del peligro que simboliza Sudán para sí mismo y para sus países limítrofes, ya que éstos reciben a un gran número de refugiados, sufren el flagelo del transporte de armas, etc. (Mendoza, 2011, p. 49).

En cuanto a Timor Oriental, se le considera como un Estado fallido porque existen temas críticos como "la injerencia extranjera en su política exterior, la crisis humanitaria referente a los refugiados, la deslegitimación del Estado y las carencias en securitización" (Cadena, 2010, p. 68). Básicamente ese ha sido un Estado donde los poderíos coloniales trataron siempre de obstaculizar la construcción de nación. Por ejemplo, Portugal arribo a ese territorio en el siglo XVI, pero nunca estuvo dentro de sus prioridades construir un Estado funcional, ya que Timor Oriental no contaba con suficientes recursos naturales y así Portugal optó más bien, por ayudar a desestructurar el país mediante el acrecentamiento de problemas étnicos. Luego de la independencia de Portugal -en la década de 1970-, Timor Oriental sufre invasión por parte de Indonesia, la cual duró casi 30 años, lo cual fue factor clave para no poder crear un Estado poscolonial, y más bien, agravó las disyuntivas culturales, sociales, políticas y económicas del país (las cuales perduran hasta el día de hoy).

Respecto a Pakistán, se debe recordar a Alberto Priego, quien postula que a pesar de que ese país haya y esté actualmente en las primeras posiciones del índice de Estado fallido de Foreign Policy, no se puede considerar como tal, sino que se le debe caracterizar como un Estado 
débil en vía de convertirse en fallido, debido a que si bien tiene problemas como terrorismo, bajo nivel de desarrollo humano, altos niveles de corrupción, etc., sus problemáticas no han desbordado la capacidad estatal, en comparación a Somalia, Sudán o el Líbano de los años 80 (Priego, 2012, p. 89).

Otro caso del que se ha escrito ha sido Guatemala. Sobre el tema, Javier Antonio Brolo, al analizar la idea de que Guatemala es un Estado fallido, ha concluido que no puede considerarse como un Estado fallido, argumentado en que si bien Guatemala

"exhibe un desempeño en sus respectivas funciones lejos de ser ejemplar y estar por debajo del promedio latinoamericano, en el año 2009 el país habría mejorado respecto al 2005 en el cumplimiento de sus principales funciones y superaba a más de un tercio de las naciones del mundo. Sin embargo, es innegable que el Estado guatemalteco debe fortalecer urgentemente las entidades responsables de cumplir las funciones vinculadas con el funcionamiento del Estado de derecho y el efectivo control del monopolio de la fuerza" (Brolo, 2010, p. 8), debido a que es claro que en ese país centroamericano, las lógicas de corrupción, seguridad, injusticia y violencia son imperantes.

Continuando con la lista aparece Argentina, el cual según algunas tesis, es un Estado fallido. Una de ellas se sustenta en la idea de que luego de la dictadura de 1.976, y de los posteriores gobiernos de los años 80 y 90, se comienza a configurar Argentina en un Estado fallido, ya que surgieron varias problemáticas a las cuales el Estado no fue capaz de responder;

"en el proceso de democratización política desarrollado desde mediados de los 80 en la Argentina, si bien resulto exitosa la reversión de la autonomía y la tutela militar sobre la vida institucional y se puso fin al ciclo de violencia que caracterizado la vida política local durante décadas, no fue posible la estructuración de una situación de seguridad pública signada por la regulación y conjuración efectiva del conjunto de los conflictos sociales vulneratorios de los derechos y libertades ciudadanas básicas de toda democracia consolidada"(Sain, 2004, p. 5).

Por eso se podría establecer que "al igual que en otras experiencias regionales, en la Argentina, la democratización política enmarcó la conformación de un Estado fallido" (Sain, 2004, p. 5).En ese país existe actualmente un constante desgobierno y un ineficiente sistema de seguridad policial y tampoco hay conocimiento ni diagnóstico para atacar la inseguridad (en lo últimos años crecieron las tasas de criminalidad; se presentó mayor número de homicidios, tentativas de homicidios, violencia, y demás).

De igual manera, han surgido diagnósticos que ven a Argentina no como un Estado fallido, sino como un Estado con la posibilidad de llegar a serlo porque "en la Argentina actual, la seguridad estratégica y la seguridad pública están ausentes" (Auel, p. 23), y por lo mismo, no es capaz de responder de manera oportuna a los nuevos retos, amenazas y peligros del mundo contemporáneo. 
Otro caso que puede citarse es el de Nigeria que a pesar de no ser considerado comúnmente como un Estado fallido, autores como John Emeka si destacan las lógicas de corrupción, rezago económico, inestabilidad política (golpes militares, fraudes electorales, guerras civiles) y violencia, situaciones que surgieron después de su independencia en el año 1960, ya que posteriormente "la violencia se convirtió en sinónimo de lucha por el poder político en todo el territorio nigeriano" (Emeka, 2007, p. 11). Por lo tanto, y como lo declara ese autor: "el Estado nigeriano ha perdido su capacidad de proporcionar seguridad consecuencia de su politización. El Estado nigeriano ha perdido la capacidad de proporcionar seguridad a sus ciudadanos y tiene aún menos capacidad para brindarles bienes políticos públicos. Por lo tanto, no es una exageración decir que el proyecto del Estado nigeriano ha fracasado" (Emeka, 2007, p. 12).

Respecto al Estado colombiano se han elaborado diferentes estudios, entre ellos el realizado por Diana Carolina Díaz, el cual parte del análisis de las posiciones de Colombia en el índice que elabora el Fondo para la Paz y Foreign Policy, llegando a la conclusión de que es casi imposible determinar si Colombia es o no un Estado fallido, solamente con base en indicadores". Lo cierto es que "en algunos aspectos- como la provisión de servicios básicos y la protección de sus ciudadanos -el Estado colombiano presenta fallas severas. Sin embargo, declararlo "fallido" hace caso omiso de una serie de avances- en materia de salud y educación, y en cuanto a la generación de ingresos- que de hecho se han logrado a través de las décadas" (Rettberg, 2010, p. 183).

Por último, respecto a lo que se ha escrito en español sobre los casos de Estados fallidos, es importante recordar el caso latinoamericano. Si bien se debe advertir lo complejo que significa analizar un caso regional por su amplitud, Laura Tedesco hace un análisis de la teoría de la falla estatal aplicada a América latina, partiendo de la siguiente tesis: "El Estado no puede ser estudiado si se lo aísla de su desarrollo histórico que comprende un largo proceso de conflictos" (Tedesco, 2007, p. 2). Por lo mismo, llega a la conclusión que los estados latinoamericanos, a pesar de su anomia no son fallidos, sino que están aún en etapa de formación, lo que conlleva a diversos procesos de conflicto y confrontación en sus fronteras.

\section{ESTADOS FALLIDOS E INTERVENCIÓN INTERNACIONAL}

Se puede observar que en las actuales discusiones respecto a los Estados fallidos, un punto neurálgico es el tema de la intervención puesto que se piensa que el mejor antídoto para las disfunciones estatales es realizar una intervención o brindar un determinado número de recetas - por parte de ciertas organizaciones internacionales o Estados-, para ayudar a aquellos países que se les considere como fallidos, débiles, colapsados, etc.; todo con el fin de que

1. Véase: Díaz, 2011. 
los mismo salgan de sus disyuntivas internas. Por ello surgen preguntas como: "¿Cuál debería ser la conducta de la comunidad internacional ante la existencia de este tipo de Estados? ¿Indiferencia o intervención?" (Mendoza, 2005, p. 85).

Acorde con los cuestionamientos acabados de mencionar y para dar respuesta a los mismos, se puede decir, como señala que el accionar internacional con respecto a los Estados fallidos, fluctúa entre la asistencia humanitaria y la intervención de fuerzas de estabilización; pero hasta la fecha ninguna de las dos ha logrado cambiar totalmente la situación de falla estatal, dado que la mayoría de los Estados fallidos continúan con sus dinámicas de anomia, a pesar del accionar de la comunidad internacional (Mendoza, 2005, p. 95).

En cuanto al panorama pro intervencionista, hay que indicar que viene anclado al paradigma liberal que promueve la comunidad internacional, por lo que en la actualidad se da una especie de consenso en pro de la intervención humanitaria y se legitiman agendas como la de la responsabilidad de proteger. Al respecto, Hideaki Shinoda comenta que "el discurso sobre la responsabilidad de proteger, como base teórica de la consolidación de la paz para los Estados fallidos, determinan cómo la sociedad internacional tiende a entender la soberanía del Estado en el siglo XXI" (Shinoda, 2010, p. 22). Shinoda se refiere que lo anterior se presenta puesto que el concepto de Estado fallido muestra los momentos críticos de la soberanía del Estado liberal; un Estado liberal que sugiere que un Estado soberano no es una entidad estable, sino que puede ser trastornada, reformada e intervenida (Shinoda, 2010, p. 21).

Cuando se plantea que las consecuencias de los Estados fallidos no solo marcan el rumbo de su dimensión interna, sino que además desestabilizan la región en que se encuentran -pudiendo llegar hasta el extremo de afectar todo el sistema internacional-, se produce un imaginario en la comunidad internacional que lleva a que a esos tipos de Estado se les observe regional y mundialmente como "focos de conflictos y guerras duraderas, desplazamiento y migraciones forzosas, crisis humanitarias, violaciones de los derechos humanos, y de nuevos tipos de amenazas como el terrorismo, el tráfico de armas y de drogas, transmisión de enfermedades y redes criminales trasnacionales" (Jaureguiberry, 2008, p. 1).

Ahora bien, gracias a las características de los sucesos y amenazas mencionadas, se puede establecer que los peligros de los Estados fallidos pueden traspasar las fronteras nacionales, y así como pueden agotarse en simples irrupciones hacia países vecinos, siempre está el miedo latente en el sistema internacional de que esos problemas alcancen una injerencia global. Es así como

"por lo general, los países tercermundistas, [los asiáticos] los africanos y los latinoamericanos, así como las ex repúblicas soviéticas son las zonas geográficas donde la comunidad internacional ha encendido sus alarmas para evitar los casos de posibles fracasos estatales. Estos países, en el contexto de la globalización, representan para sus vecinos y para la comunidad internacional una grave amenaza por los efectos de su desintegración" (Martelo, 2009, p. 72). 
Por su parte, otra cuestión de suma importancia en lo que se refiere a Estados fallidos e intervención internacional, es que los Estados fallidos representan una amenaza para el Derecho Internacional Humanitario y presentan polémicas frente al Derecho Internacional.

Si bien es difícil determinar qué normas exactamente del D.I.H. se violan en los Estados fallidos, sí puede postularse de manera genérica, que la falla estatal conlleva problemas para el D.I.H. debido a factores-para mencionar algunos casos- como: "la desaparición de mandos responsables, proliferación de armas pequeñas y ligeras entre facciones confusas y difíciles de identificar, [y] la implicación de los niños en los conflictos armados como combatientes" (Pozo, 2004, p. 10). Ello tiene como consecuencia una preocupación constante de varias organizaciones no gubernamentales, Estados, etc.; generando con ello una justificación perfecta para la intervención en los Estados con disfuncionalidades internas. Citando a Pilar Pozo "[en los Estados fallidos] se producen violaciones masivas y graves de derechos humanos, cuestión que concierne a la comunidad internacional que ha podido invocar así la protección de los derechos humanos o el principio de libre determinación de los pueblos como titulo legitimador de la intervención" (Pozo, 2004, p. 7).

En lo que corresponde al D. I.H., es obligatorio traer a colación a Ana Gemma López, ya que la autora plantea que más que el D. I. H o los Derechos Humanos, los Estados fallidos reflejan una problemática para el Derecho Internacional en general porque en ese derecho solamente existe la figura del Estado.; entonces los Estados fallidos y demás tipologías estatales son inexistentes en la normatividad jurídica internacional. Y como lo declara la autora, los Estados fallidos representan "una realidad que tiene que ver con el propio funcionamiento del Estados como tal en el marco del ordenamiento jurídico internacional, con su imposibilidad de ejercer plenamente su faceta como sujeto pleno de Derecho Internacional. Y estos se traduce en numerosas complicaciones" (López, 2010, p. 5).

Para finalizar, entre las diversas formas de responder a las consecuencias de los Estados fallidos por parte de los actores externos -y desde la literatura escrita en español- llama la atención el enfoque de análisis que se denomina "nation-building" o la construcción de nación que es básicamente, según Agustin Jaureguiberry: "la necesidad de implementar, por parte de la comunidad internacional, acciones y planes tendientes a fortalecer las intenciones y la capacidad del Estado [...] Para que así puedan contar con las herramientas que prevengan el surgimiento o reaparición de los problemas anteriormente descritos" (Jaureguiberry, 2008, p. 1).Cabe destacar que este tipo de ideas -si bien son interesantes- se hacen realmente complejas de implementar porque una construcción de nación desde afuera y con lineamientos top-down, desconoce y en ocasiones ignora, las condiciones y lógicas que tienen los personajes e instituciones locales.

\subsection{INSTRUMENTALIZACIÓN CONCEPTUAL}

En gran parte y gracias a la creciente preocupación de la comunidad internacional hacia los Estados fallidos, ha ido creciendo considerablemente el tema de la politización e 
instrumentalización del concepto. Sobre el tema, Flórez señala que los primeros años del siglo XXI trajeron consigo un punto de inflexión en el que hacer de la teoría de la falla estatal, al dejar de concebir a los Estados fallidos como una preocupación humanitaria -como solía hacerse en la década de los años 90- y comenzar a verlos como una amenaza para la seguridad planetaria (debido a que pueden ser nidos de criminalidad transnacional, grupos terroristas, producción de armas de destrucción masiva, guerras internas, desplazamiento forzado transnacional, desestabilización regional) (Flórez, 2011, p. 196). Así, el problema en cuestión pasó a tener una mayor preocupación tanto en la agenda política internacional como en la agenda nacional de algunos Estados, lo que inmediatamente trajo algunas consecuencias. Siguiendo a José Flórez "el resultado inmediato de la entrada de los estados fallidos en la agenda diplomática y de seguridad internacional fue la politización [e instrumentalización] del debate académico" (Flórez, 2011, p. 196).

En este tema de la politización es interesante el punto de partida del profesor Juan Gabriel Tokatlian, quien señala que un ejemplo de esta problemática se evidencia en las relaciones bilaterales entre Colombia y Estados Unidos: "las relaciones de Washington y Bogotá desde mediados de los noventa en adelante [pueden ser utilizadas] como un estudio de caso en el que se muestra la forma en que la construcción en torno a la cuestión del Estado Fallido fue esencial para legitimar una creciente y masiva intervención estadounidense en los asuntos colombianos" (Tokatlian, 2008, p. 68). Es decir, en este caso se utilizó el concepto de Estafo fallido de manera instrumental por parte de Estados Unidos, para intervenir de diversas formas en Colombia.

Por la misma línea, Tokatlian reitera la idea de que, si bien el Estados fallido nació en la academia, fue llegando gradualmente al gobierno estadounidense, y con ello se fue estableciendo cierto tipo de "comunidad científica a sui generis", acorde con los intereses gubernamentales de ciertos Estados, y por lo mismo, la noción de falla estatal se empezó a utilizar para legitimar el accionar internacional estadounidense y de otros Estados (Tokatlian, 2008, p. 68). Así, los estados fallidos -más que mantenerse como objetos de estudio que se debían analizar y estudiar desde la academia-, fueron pasando a las agendas gubernamentales de Estados Unidos y otros actores internacionales.

Por otro lado, retomando el punto de inflexión que se dio a principios de la década del 2000,

“la reteorización de la retórica y la política después de los atentados terroristas del 9/11 llevó a que se necesitara conceptualizar y teorizar acerca de este fenómeno, no sólo relacionando causas y efectos, sino que yendo aún más lejos, sentando juicios de valor y una posición clara en cómo debería actuar los Estados y las organizaciones multilaterales en estos escenarios" (Monroy, 2011, p. 19).

Ello dio como resultado que se relacionara directamente la falla estatal y cuestiones como el terrorismo internacional, generando así que la teoría de los Estados fallidos no fuera tanto una 
descripción académica de ciertos Estados con fallas, sino más bien un accionar político, militar y económico contra dichos Estados. En otros términos, el Estado fallido paso de ser una teoría científica a una retórica política en pro de determinados intereses.

Un claro ejemplo del nivel en que se ha politizado e instrumentalizado la teoría de los Estados fallidos es que, "poco antes de acceder a la presidencia, Barack Obama- en la ceremonia de presentación del equipo que coordinaría la comunidad de inteligencia durante su administraciónse refirió a los grades retos de seguridad de su país. Destaco dos: Los estados Fallidos y el terrorismo internacional" (Gutiérrez, 2009, p. 88); además la frase, como "Colombia: de Estado Fracasado a estrella emergente", fue escuchada en la boca de mandatarios colombianos como Álvaro Uribe y Juan Manuel Santos. De hecho, Lawrence Summers llegó al extremo de afirmar que "Colombia ha pasado de ser un Estado Fallido a convertirse en un Estado que es hoy modelo internacional" (Flórez, 2011, p. 198).

A modo de conclusión, se puede establecer que el fenómeno de la falla estatal ya no se remite únicamente a las aulas de clase o centros de investigación académicos. Por ejemplo, es claro que los índices de Estados fallidos se han ido consolidando como generadores de opinión pública tanto a nivel local como internacional, a favor de determinados intereses (Flórez, 2011, p. 197). Por lo anterior, y sumado a cuestiones como que el Estado fallido puede ser utilizado como discurso político, y así mismo, se ha convertido en una pieza clave en las agendas gubernamentales para cuestiones como la intervención internacional y la erradicación del terrorismo global, puede decirse que el Estado fallido ha ido mutando de objeto de estudio académico hacia las esferas políticas, y poco a poco, se ha convertido en un instrumento discursivo más que explicativo, por lo mismo, las acciones de varios Estados,

"no sólo de los Estados Unidos, tanto a nivel interno como externo está atado a esta temática [de los Estados fallidos]. Ya sea como Estados fuertes que buscan proveer soluciones a la debilidad de los Estados en función de intereses particulares o como Estados débiles actuando en función de mejorar sus indicadores de seguridad, políticos, económicos y sociales para no caer en la categoría de Estados fallidos o débiles. Este hecho muestra el alcance que ha tenido este discurso y su aceptación por distintos actores (Monroy, 2011, p. 3)

\section{CONCLUSIONES}

Luego de observar los puntos más problemáticos y que generan más disensos en la literatura especializada de la teoría del Estado fallido, se puede plantear que hay unos puntos polémicos en lo que respecta primeramente a la definición. Se ha tratado de mostrar que no existe una univoca definición de lo que se entiende por Estado fallido. Es por eso que la mayoría de autores especializados en el tema, cuentan cada uno con su propia definición. Sin embargo, a pesar de que no existe consenso, se pueden ubicar ciertas tendencias de conceptualización en escuelas; las que básicamente son dos: una conservadora y una crítica. 
A pesar del debate que genera la definición del Estado fallido podría decirse que un Estado es fallido o comienza a fallar cuando las estructuras internas no son viables, y por ende, el aparato se hace disfuncional y se complejiza al momento de responder a todas las demandas de la ciudadanía; llevando a que los bienes tanto políticos como sociales, económicos y culturales, se vean deteriorados. Así, se dirá que un Estado falla cuando otros agentes le disputan el monopolio de la violencia, las condiciones de inseguridad se establecen como un común denominador, las políticas sociales se hacen ineficaces para responder a las demandas de la comunidad, el régimen político se degenera y deja de representar o servir a las personas, la economía es obsoleta y genera todo tipo de crisis, el desarrollo humano y la seguridad humana no se garantizan o son precarios, entre otros.

Del mismo modo, hay que recordar que existe una problemática de sinonimia en cuanto a otras topologías estatales (Estados criminales, Estados débiles, cuasi Estados, y otros) que se suelen relacionar y utilizar indiscriminadamente en relación al Estado fallido. Pero se debe advertir, que el Estado fallido es una teoría propia que se viene trabajando hace más de dos décadas, y tiene su propia aglomeración teórica.

Por otra parte, y otro tema que genera un gran debate en la teoría de la falla estatal, es la metodología utilizada para medir y dictaminar a los Estados fallidos. El método por excelencia para establecer qué Estado es fallido, es el cuantitativo, ya que en la última década se ha dado una proliferación de índices de Estados fallidos; pero tales índices tienen una serie de críticas, tales como su ahistoricidad, sus variables, y demás. A pesar de la serie de objeciones que desde la literatura especializada se han puesto, vale la pena advertir que son valiosos esfuerzos para saber qué Estados están en una situación de fracaso, así como la intensidad de anomia que sufren; por lo que si bien se deben analizar con cautela, son un referente importante en el tema.

En este orden de ideas, se hace importante mencionar que en los trabajos académicos, en español, también se han ido elaborando análisis de metodologías cualitativas, expresadas en estudios de caso, que evidencian el estado de falla y fracaso de ciertos Estados. Es por eso que se han elaborado escritos para trabajar a países como Somalia, Sudan, Pakistán, Argentina, para mencionar algunos casos.

Para terminar, y entrando al último tema que se trabajó en el texto, hay que decir que los Estados fallidos están directamente relacionados con la intervención internacional, ya que la sociedad internacional entra en constantes dilemas de qué hacer con los mismos, debido a que se presume que dichos Estados no representan sólo un problema interno, sino que también lo son para la región en que se encuentran y el resto del mundo. Es por ello que se han practicado y se practican intervenciones internacionales (humanitarias y militares), para salvar a los Estados fallidos; porque en ellos se violan los Derechos Humanos y el Derecho Internacional Humanitario. Sin embargo, las consecuencias de tales actos no tienen los mejores resultados. Adicionalmente, esas intervenciones han llevado, en parte, a 
que se politice o instrumentalice la noción de Estado fallido, y se use más en los medios de comunicación y en las esferas políticas; también que se aleje cada vez más de la académica, por lo que se hace necesario retomar desde la investigación científica, la teoría del Estado fallido, porque dicha teoría puede ayudar a entender adecuadamente ciertos Estados que están afectados por diversas problemáticas.

\section{REFERENCIAS}

- Auel, Federico. La Argentina y su probabilidad de llegar a la situación de Estado Fallido como riesgo estratégico, en el mediano plazo. Manuscrito no publicado. Buenos Aires, Argentina: Universidad Maimonides.

- Alcalde, Javier (2004). Los Estados Fallidos: la influencia del desarrollo. Lima: CEDEP.

- Álvarez, Juan (2007). La etiqueta como estigmatización: De Failed State, a Rouge State. Revista electrónica de estudios internacionales, (14). Disponible en: http://www.reei.org/ index.php/revista/num14/notas/etiqueta-como-estigmatizacion-failed-state-rogue-state [consultado 3 de septiembre, 2012]

- Brolo, Javier Antonio (2010). Guatemala no es un Estado Fallido. Un estudio sobre el desempeño de las instituciones del Estado guatemalteco en diez funciones fundamentales. Revista ASIES, (3). Disponible en: http://www.kas.de/wf/doc/2316-1442-4-30.pdf [consultado 15 de septiembre, 2012].

- Cadena, W. Devia, C. (2010).Timor Este o la construcción de la nación en un Estado Fallido. Revista de relaciones internacionales, estrategia y seguridad. 5 (2), pp.:39-69.

- Castellón Joaquín (2011). Somalia: el paradigma del Estado Fallido. Panorama geopolítico de los conflictos 2011. España: Ministerio de Defensa y Dirección General de Relaciones Institucionales.pp.:136-166

- Chomsky, Noam (2007). Estados Fallidos: el abuso de poder y el ataque a la democracia. Barcelona: Ediciones B.

- Díaz, Diana (2011) Discusión de las teorías de Estado Fallido: Ilustración Colombia durante la administración del presidente Uribe Vélez (2002-2010). Manuscrito no publicado. Bogotá, Colombia. Universidad Colegio Mayor de Nuestra Señora del Rosario.

- Di John, Jonathan (2010). Conceptualización de las causas y consecuencias de los Estados Fallidos: una reseña crítica. Revista de Estudios Sociales. (37), pp.:46-86. 
- Doornbos, Martín (2011). ¿Cuándo un Estado es un Estado? Explorando Putlandia, Somalia. Relaciones Internacionales, (18). Disponible en: http://www.relacionesinternacionales. info/ojs/article/view/315.html [Consultado 10 septiembre, 2012].

- Duffield, Mark (2008). Los estados frágiles y el retorno de la administración nativa. Revista Académica de Relaciones Internacionales, (8). Disponible en: http://www. relacionesinternacionales.info/ojs/article/view/105.html [Consultado 10 septiembre, 2012].

- Emeka, John (2007). Fracaso y colapso del Estado africano: el ejemplo de Nigeria. FRIDE. Disponible en: http://www.fride.org/download/akude.esp.pdf\%E2\%80\%8E [consultado 10 de septiembre, 2012].

- Fernández, Encarnación (2009). ¿Estados Fallidos o Estados en Crisis? Granada: Editorial Colmenares.

- Flórez, José (2011). ¿Estado o concepto fallido? Problemas que plantean la noción de falla estatal y los índices que intentan medirla. Revista del derecho del Estado. (27), pp.: 198234.

- Freddyur, Luis (2009). Estados Fallidos o por colapsar. Criterio Jurídico. 9 (1), pp.: 55-88.

- Gonzales, Fernán; Rettberg, Angeliza (2010). Pensando la fragilidad estatal. Colombia. Revista de Estudios Sociales. (37), pp.: 181-184.

- Gutiérrez, Francisco (2010).zEstados Fallidos o conceptos Fallidos? La clasificación de las fallas estatales y sus problemas. Revista de Estudios sociales, (37), pp.: 87 - 104.

- Jaureguiberry, Agustin (2008). Los Estados Fallidos y el fenómeno del nation-biuilding. Trabajos y ensayos, (.8). Disponible en: http://www.diprriihd.ehu.es/revistadoctorado/n8/ Jaureguiberry08.pdf [consultado 10 de septiembre, 2012].

- Jackson, Roberth (2009).Los Estados Fallidos y la tutela internacional. Revista Académica de Relaciones Internacionales, (10). Disponible en: http://www.relacionesinternacionales. info/ojs/issue/view/10.html [consultado 10 de septiembre, 2012].

- López, Ana (2010). Los Estados Fallidos y sus implicaciones en el ordenamiento jurídico internacional. Cursos de derecho internacional y relaciones internacionales de vitoriaGasteiz (pp.:159-240). Bilbao: Servicio de publicaciones de la Universidad del País Vasco.

- López, Roman, Almazán Marco (2009). Somalia. Estado fallido y piratería marítima. Estudios de Asia y África. XLIV (3), pp.: 583- 607. 
- Martelo, Catalina (2009) Los Estados Fallidos en el contexto de la pérdida de hegemonía de occidente. Manuscrito no publicado. Medellín: Universidad de Antioquia.

- Mendoza, Daniela (2011). Análisis de la perpetuación del conflicto interno sudanés bajo la categoría de Estado Fallido, tras la firma del acuerdo de paz global de 2005. Manuscrito no publicado. Bogotá: Universidad Colegio de Nuestra Señora del Rosario.

- Mendoza, Juan (2005) Los Estados Fallidos- Estados desestructuradas: una amenaza a la seguridad internacional o un pretexto para la injerencia de las grandes potencias. Revista política y seguridad. (98).Disponible en: http://www.anepe.cl/wp-content/uploads/Anepe98-COMPLETA.pdf [consultado 16 de diciembre, 2012].

- Monroy, Santiago (2011) Análisis de la construcción del concepto de Estado Fallido y su vinculación con la agenda política estadounidense como forma legitimar la acción política internacional. Manuscrito no publicado. Bogotá, D.C. Universidad Colegio Mayor de Nuestra Señora del Rosario.

- $\quad$ Priego, Alberto (2012). Pakistán: Un Estado Fallido, débil o colapsado. UNISCI Discussion Papers, (29), pp.: 67-89.

- Pozo, Pilar (2004). Estados fallidos, derecho internacional humanitario y seguridad internacional. Los retos humanitarios del siglo XXI (pp. 173- 185). Valencia: C ramón Chornet.

- Rojas, Diana (2007). La falla estatal y la globalización. Análisis político. (61), pp.: $73-85$

- $\quad$ Robert, Rotberg, Clapham, Chistopher, Herbst, Jeffrey (2007). Los Estados Fallidos o Fracasados: Un debate inconcluso y sospechoso. Estudio preliminar, Patricia Moncada. Bogotá,D.C.: Siglo del Hombre editores.

- $\quad$ Sain, Marcelo (2004). Un Estado Fallido en las nuevas problematices delictivas. El caso argentino. Universidad de Belgrado .Disponible en: http://repositorio.ub.edu.ar:8080/ xmlui/handle/123456789/283 [consultado 10 de enero, 2013].

- $\quad$ Salas, Cristian (2011). Estados débiles, espacios sin ley e ingobernabilidad. Disponible en: http://www.orpas.cl/ [consultado 30 de septiembre, 2012].

- $\quad$ Shinoda, Hedeaki (2010). Relaciones entre la teoría contemporánea del Estado soberano y la consolidación de la paz. CS, (3). Disponible en: http://bibliotecadigital.icesi.edu.co/ biblioteca_digital/handle/10906/2160 [consultado 20 de septiembre, 2012]

- Tokatlian, Gabriel (2008). La construcción de un Estado Fallidos en la política mundial: el caso de las relaciones entre Estados Unidos y Colombia. Análisis político. (64), pp.: 67-104. 
- Vilanova, Pere (2008). ¿Estados de facto versus fallidos o frágiles? Unas notas acerca de la dificultad teórica para establecer nuevas tipologías. Revista académica de relaciones internacionales. (10). Disponible en: http://www.relacionesinternacionales.info/ojs/article/ view/141.html [consultado 10 de septiembre, 2012]

- Weber, Max (1992). La ciencia como profesión La política como profesión. Madrid: Editorial Espasa Calpe.

- Woodward, Susan, Taylor, Mark (2005). Estados Frágiles: soberanía, desarrollo y conflicto. Madrid: Centro de investigación para la paz (CIP-FUHEM).

- Ylönen, Aleksi (2008). Conflicto y crecimiento: la configuración y supervivencia del estado fallido en Sudán. Revista Académica de Relaciones Internacionales. (8). Disponible en: http://www.relacionesinternacionales.info/ojs/article/view/107.html [consultado 15 de septiembre, 2012]. 